\title{
Third Order ODEs Systems and Its Characteristic Connections
}

\author{
Alexandr MEDVEDEV \\ Faculty of Applied Mathematics, Belarusian State University, \\ 4, Nezavisimosti Ave., 220030, Minsk, Republic of Belarus \\ E-mail: Sasha.Medvedev@gmail.com
}

Received April 20, 2011, in final form July 27, 2011; Published online August 03, 2011 doi:10.3842/SIGMA.2011.076

\begin{abstract}
We compute the characteristic Cartan connection associated with a system of third order ODEs. Our connection is different from Tanaka normal one, but still is uniquely associated with the system of third order ODEs. This allows us to find all fundamental invariants of a system of third order ODEs and, in particular, determine when a system of third order ODEs is trivializable. As application differential invariants of equations on circles in $\mathbb{R}^{n}$ are computed.
\end{abstract}

Key words: geometry of ordinary differential equations; normal Cartan connections

2010 Mathematics Subject Classification: 34A26; 53B15

\section{Introduction}

\subsection{Differential equation as a structure on a filtered manifold}

The main purpose of this article is to study geometry of systems of ordinary differential equations of third order. The geometry of ordinary differential equations or, more generally, of differential equation of finite type is based on the general theory of geometric structures on filtered manifolds. First it was developed by Tanaka in [9, 10]. Recall that a filtered manifold is a smooth manifold $M$ equipped with a filtration of the tangent bundle $T M$ compatible with the Lie bracket of vector fields. At any point $x \in M$ the associated graded vector space $\operatorname{gr} T_{x} M$ can be endowed with a Lie algebra structure. This nilpotent Lie algebra $\mathfrak{m}$ is called a symbol of a filtered manifold (at the point $x$ ). In the paper we consider only the so-called filtered manifolds of constant type, assuming that the graded nilpotent Lie algebras $\operatorname{gr} T_{x} M$ are isomorphic to each other for all points $x \in M$.

By a symbol of a geometric structure on $M$ we understand a graded Lie algebra $\mathfrak{g}$ with the negative part $\mathfrak{g}_{-}=\sum_{i<0} \mathfrak{g}_{i}$ which is equal to the symbol $\mathfrak{m}$ of the filtered manifold $M$ of constant type. Here the Lie algebra $\mathfrak{m}$ is the subalgebra of a so-called universal Tanaka prolongation $\mathfrak{g}(\mathfrak{m})$. Roughly speaking, this means that $\mathfrak{g}(\mathfrak{m})$ is the maximum among graded Lie algebras which satisfy the condition "for any element $X \in \mathfrak{g}_{i}, i \geq 0$ the equality $\left[X, \mathfrak{g}_{-}\right]=0$ implies $X=0$ ".

An arbitrary equation $\mathcal{E}$ can be viewed as a surface in jet space. The canonical restriction of the contact distribution on jet space defines the structure of filtered manifold on $\mathcal{E}$.

\subsection{The problem of equivalence}

One of the main problems in the theory of differential equations is the problem of equivalence. Two differential equations are called equivalent if one can be transformed to another by a certain 
change of variables. We consider equations up to point transformations, i.e. we allow arbitrary changes of both dependent and independent variables.

First classical approach to the equivalence problem of ODEs was developed by Sophus Lie. In [5] he obtains partial results about second order ODEs. The complete answer was given later by Tresse [11]. Invariants of the third order ODEs were computed by Chern in his paper [1]. A modern approach to the equivalence problem of ODEs can be found in the papers [2] and [4], where characteristic Cartan connection was constructed for the one equation of arbitrary order and for the system of ODEs of the second order.

The general approach to the equivalence problem for the holonomic differential equations can be find in [3]. The key fact there is the existence of a full functor from the category of holonomic differential equations to the category of Cartan connections. This reduces the equivalence problem for differential equations to the equivalence problem for the corresponding Cartan connections.

\subsection{Normalization of Cartan connections}

Let $P$ be the principal $H$-bundle. Let $\omega$ be a Cartan connection of type $(G, H)$, where $G$ is a Lie group with a semisimple graded Lie algebra $\mathfrak{g}$ and $H$ is a parabolic subgroup of $G$ with the Lie algebra $\mathfrak{h}$. In the paper [10] Tanaka built a set of normal Cartan connections on the principal bundle $P$ as follows. He used the scalar product defined with the help of the Killing form to construct adjoint Lie algebra codifferential $\partial^{*}$. Then a Cartan connection is normal iff the structure function $C: P \rightarrow \operatorname{Hom}\left(\wedge^{2} \mathfrak{g}_{-}, \mathfrak{g}\right)$ belongs to the kernel of the operator $\partial^{*}$ and the structure function has not negative components. As usual define a Laplacian $\Delta=\partial^{*} \partial+\partial \partial^{*}$. The structure function $C$ decomposes as $C=H(C)+\Delta(C)$. The component $H(C)$ is called the harmonic part of the structure function. The key fact about it is that $H(C)$ is the fundamental system of invariants (see Definition 5 for details). In the case of the geometry of holonomic differential equations the Lie algebra $\mathfrak{g}$ is not necessarily semisimple. However in [3] is shown that we still can find the scalar product on $\mathfrak{g}$ such that the normal Tanaka conditions define the unique Cartan connection associated to a holonomic differential equation.

In this paper we associate with every system of ODEs of third order a characteristic Cartan connection which differ from a normal Tanaka Cartan connection. The reason for doing this is a relation between conformal geometry and geometry of the system of the third order ODEs. Conformal manifold is determined by the family of conformal circles, which was shown by Yano [12]. Each conformal circle is determined by the point on it, the direction and the curvature, i.e. by the point in the third jet space. The system of appropriate differential equations of the third order gives us the bridge between the conformal geometry and the geometry of the differential equation. It is appeared that a characteristic Cartan connection, which is built in the paper, is in close relations with the normal conformal Cartan connection. The relation of the conformal geometry and the geometry of third order ODEs is the topic of the next paper.

The paper is organized as follows. In Section 2 we naturally associate the system of the third order ODEs with the pair of distributions. This pair of distributions give rise to the filtered manifold associated with the system of the third order ODEs. We write down the symbol of the system of ODEs of the third order, the notion of adopted coframe and adopted Cartan connection. The problem of equivalence is considered in Section 3. When we working in the case of semisimple Lie algebras and normal Cartan connections, the harmonic part of the curvature gives us the fundamental system of differential invariants. We show that in general case fundamental differential invariants are contained in the Ker $\partial$ part of the curvature, where $\partial$ is the Lie algebra cohomology differential. In Section 4 we build the characteristic Cartan connection uniquely associated to the the system of ODEs of the third order. This connection allows us to obtain the results about equivalence third order equations and to describe the 
structure of the fundamental invariants of the system of third order ODEs. In particular, this answers the question "When is the given system trivializable?" explicitly.

\section{Geometry of the systems of third order ODEs}

Consider an arbitrary system of $m$ ordinary differential equations of third order:

$$
y_{i}^{\prime \prime \prime}(x)=f_{i}\left(y_{j}^{\prime \prime}(x), y_{k}^{\prime}(x), y_{l}(x), x\right),
$$

where $i, j, k, l=1, \ldots, m$ and $m \geq 2$.

We associate a filtered manifold with this system in the following way. Let $J^{3}\left(\mathbb{R}^{m+1}, 1\right)$ be the third jet space of unparametrized curves. Then the equations (1) can be considered as a submanifold $\mathcal{E}$ in $J^{3}\left(\mathbb{R}^{m+1}, 1\right)$. We introduce the following coordinate system on the surface $\mathcal{E}$ :

$$
\left(x, y_{1}, \ldots, y_{m}, p_{1}=y_{1}^{\prime}, \ldots, p_{m}=y_{m}^{\prime}, q_{1}=y_{1}^{\prime \prime}, \ldots, q_{m}=y_{m}^{\prime \prime}\right) .
$$

There is a natural one-dimensional distribution $E$ whose integral curves are the lifts of solutions of equations (1). Let $\pi_{1}^{2}$ be the canonical projection from the surface $\mathcal{E}$ to the first jet space $J^{1}\left(\mathbb{R}^{m+1}, 1\right)$. We denote a kernel of a differential $\mathrm{d} \pi_{1}^{2}$ as $V$. In coordinates distributions $E, V$ have the form:

$$
E=\left\langle\frac{\partial}{\partial x}+p_{i} \frac{\partial}{\partial y_{i}}+q_{i} \frac{\partial}{\partial p_{i}}+f^{i} \frac{\partial}{\partial q_{i}}\right\rangle, \quad V=\left\langle\frac{\partial}{\partial q_{i}}\right\rangle
$$

where $i, j=1, \ldots, m$.

Define a distribution $C$ as the direct sum of the distributions $E$ and $V$. Then $C$ and its subsequent brackets define a filtration of a tangent bundle $T \mathcal{E}$ :

$$
C=C^{-1} \subset C^{-2} \subset C^{-3}=T \mathcal{E},
$$

where $C^{-i-1}=C^{-i}+\left[C^{-i}, C^{-1}\right]$.

It is easy to see that the symbol of the filtrated manifold $\mathcal{E}$ is a nilpotent Lie algebra $\mathfrak{m}$ isomorphic to the Lie algebra of vector fields generated by

$$
\mathfrak{m}_{-1}=\left\langle\frac{\partial}{\partial x}+p_{j} \frac{\partial}{\partial y_{j}}+q_{j} \frac{\partial}{\partial p_{j}}, \frac{\partial}{\partial q_{i}}\right\rangle .
$$

Let $\operatorname{Aut}_{0}(\mathfrak{m})$ be a subgroup of grading preserving elements of the group $\operatorname{Aut}(\mathfrak{m})$. The elements of the group $\operatorname{Aut}_{0}(\mathfrak{m})$ which preserve the splitting $E \oplus V$ form subgroup $G_{0}$. So the splitting $E \oplus V$ of the distribution $C$ defines $G_{0}$-structure of type $\mathfrak{m}$. The action of the group $G_{0}$ on $\mathfrak{m}$ is completely determined by its action on $\mathfrak{m}_{-1}$. The latter has the following form in the basis $\left\{\frac{\partial}{\partial x}+p_{j} \frac{\partial}{\partial y_{j}}+q_{j} \frac{\partial}{\partial p_{j}}, \frac{\partial}{\partial q_{i}}\right\}$ :

$$
\left(\begin{array}{cc}
a & 0 \\
0 & B
\end{array}\right), \quad a \in \mathbb{R}^{*}, \quad B \in G L_{m}(\mathbb{R}) .
$$

The symbol $\mathfrak{g}$ is the universal Tanaka prolongation of the pair $\left(\mathfrak{m}, \mathfrak{g}_{0}\right)$. It has the following form:

$$
\mathfrak{g}=\left(\mathfrak{s} l_{2}(\mathbb{R}) \times \mathfrak{g l}_{m}(\mathbb{R})\right) \curlywedge\left(V_{2} \otimes W\right) .
$$

In other words, $\mathfrak{g}$ is equal to the semidirect product of the Lie algebra $\mathfrak{s l} l_{2}(\mathbb{R}) \times \mathfrak{g l}_{m}(\mathbb{R})$ and an Abelian ideal $V$. The ideal $V$ has the form $V_{2} \otimes W$, where $V_{2}$ is an irreducible $\mathfrak{s} l_{2}$-module of dimension 3 and $W=\mathbb{R}^{m}$ is the standard representation of $\mathfrak{g l}_{m}(\mathbb{R})$. 
Let us fix a basis of the Lie algebra $\mathfrak{s} l_{2}$ and $\mathfrak{s} l_{2}$-module $V_{2}$. Let $x, y, h$ be the standard basis of an algebra $\mathfrak{s} l_{2}$ with relations:

$$
[x, y]=h, \quad[h, x]=2 x, \quad[h, y]=-2 y .
$$

This basis can be represented in the following way:

$$
x=\left(\begin{array}{cc}
0 & 1 \\
0 & 0
\end{array}\right), \quad h=\left(\begin{array}{cc}
1 & 0 \\
0 & -1
\end{array}\right), \quad y=\left(\begin{array}{ll}
0 & 0 \\
1 & 0
\end{array}\right) .
$$

Let $v_{0}, v_{1}, v_{2}$ be a basis of the module $V_{2}$ such that $x \cdot v_{2}=v_{1}, x \cdot v_{1}=v_{0}, x \cdot v_{0}=0$.

Define the grading of the Lie algebra $\mathfrak{g}$ as follows:

$$
\begin{aligned}
& \mathfrak{g}_{1}=\langle y\rangle, \quad \mathfrak{g}_{0}=\left\langle h, \mathfrak{g l}_{m}\right\rangle, \quad \mathfrak{g}_{-1}=\langle x\rangle+\left\langle v_{2} \otimes W\right\rangle, \\
& \mathfrak{g}_{-2}=\left\langle v_{1} \otimes W\right\rangle, \quad \mathfrak{g}_{-3}=\left\langle v_{0} \otimes W\right\rangle .
\end{aligned}
$$

To build a natural Cartan geometry associated to the equation (1) we will use the fact [7] that under some additional conditions (which are satisfied for geometric structures arising from holonomic differential equations, see [3]) there exists a full functor from the category of $G_{0^{-}}$ structures of type $\mathfrak{m}$ to the category of Cartan connections of type $(G, H)$, where $G$ and $H$ are the Lie groups with Lie algebras $\mathfrak{g}$ and $\mathfrak{h}$ respectively which are determined from $G_{0}$ in natural manner. The group $G$ is a semisimple product:

$$
G=\left(S L_{2}(\mathbb{R}) \times G L_{m}(\mathbb{R})\right) \curlywedge\left(V_{2} \otimes W\right),
$$

and the group $H$ is the following subgroup of $G$ :

$$
H=\left(\begin{array}{cc}
a & b \\
0 & a^{-1}
\end{array}\right) \times A, \quad a \in \mathbb{R}^{*}, \quad b \in \mathbb{R}, \quad A \in G L_{m}(\mathbb{R}) .
$$

Note that the corresponding subalgebra $\mathfrak{h}$ is exactly the nonnegative part of the Lie algebra $\mathfrak{g}$ :

$$
\mathfrak{h}=\sum_{i \geq 0} \mathfrak{g}_{i}
$$

Definition 1. We say that a coframe $\left\{\omega_{-3}^{i}, \omega_{-2}^{i}, \omega_{-1}^{i}, \omega_{x}\right\}$ on $\mathcal{E}$ is adapted to equation (1) if:

- the annihilator of forms $\omega_{-3}^{i}, \omega_{-2}^{i}, \omega_{x}$ is $V$;

- the annihilator of forms $\omega_{-3}^{i}, \omega_{-2}^{i}, \omega_{-1}^{i}$ is $E$;

- the annihilator of forms $\omega_{-3}^{i}$ is $C^{-2}$.

Let $\bar{\pi}: P \rightarrow \mathcal{E}$ be a principle $H$-bundle and let $\bar{\omega}$ be and arbitrary Cartan connection of type $(G, H)$ on $P$. Connection $\bar{\omega}$ can be written as:

$$
\bar{\omega}=\bar{\omega}_{-3}^{i} v_{0} \otimes e_{i}+\bar{\omega}_{-2}^{i} v_{1} \otimes e_{i}+\bar{\omega}_{-1}^{i} v_{2} \otimes e_{i}+\bar{\omega}_{x} x+\bar{\omega}_{h} h+\bar{\omega}_{j}^{i} e_{i}^{j}+\bar{\omega}_{y} y .
$$

Definition 2. We say that a Cartan connection $\bar{\omega}$ on a principal $H$-bundle $\bar{\pi}$ is adapted to equations (1), if for any local section $s$ of $\bar{\pi}$ the set

$$
\left\{s^{*} \bar{\omega}_{x}, s^{*} \bar{\omega}_{-1}^{i}, s^{*} \bar{\omega}_{-2}^{i}, s^{*} \bar{\omega}_{-3}^{i}\right\}
$$

is an adapted co-frame on $\mathcal{E}$.

We have described the set of Cartan connection adapted to the system of third order ODEs. However, we can chose the representative in different ways. The next two sections are devoted to the building of a canonical connection which we call characteristic. 


\section{Characteristic Cartan connection and fundamental differential invariants}

As in Section 2 let $\bar{\pi}: P \rightarrow \mathcal{E}$ be a principle $H$-bundle and let $\bar{\omega}$ be and arbitrary Cartan connection of type $(G, H)$ on $P$ :

$$
\bar{\omega}=\bar{\omega}_{-3}^{i} v_{0} \otimes e_{i}+\bar{\omega}_{-2}^{i} v_{1} \otimes e_{i}+\bar{\omega}_{-1}^{i} v_{2} \otimes e_{i}+\bar{\omega}_{x} x+\bar{\omega}_{h} h+\bar{\omega}_{j}^{i} e_{i}^{j}+\bar{\omega}_{y} y .
$$

Let $\bar{\Omega}=\mathrm{d} \bar{\omega}+\frac{1}{2}[\bar{\omega}, \bar{\omega}]$ be the curvature of the Cartan connection $\bar{\omega}$ :

$$
\bar{\Omega}=\bar{\Omega}_{-3}^{i} v_{0} \otimes e_{i}+\bar{\Omega}_{-2}^{i} v_{1} \otimes e_{i}+\bar{\Omega}_{-1}^{i} v_{2} \otimes e_{i}+\bar{\Omega}_{x} x+\bar{\Omega}_{h} h+\bar{\Omega}_{j}^{i} e_{i}^{j}+\bar{\Omega}_{y} y .
$$

Definition 3. The structure function of a Cartan connection $\omega$ is a function

$$
C: P \rightarrow \operatorname{Hom}\left(\wedge^{2} \mathfrak{g}_{-}, \mathfrak{g}\right),
$$

which is defined by

$$
C(p)\left(g_{1}, g_{2}\right)=\Omega_{p}\left(\omega_{p}^{-1}\left(g_{1}\right), \omega_{p}^{-1}\left(g_{2}\right)\right) .
$$

We can obtain the structure function of a Cartan connection explicitly. Let $\left\{e_{1}, \ldots, e_{n+k}\right\}$ be a basis of Lie algebra $\mathfrak{g}$ such that $\left\{e_{n+1}, \ldots, e_{n+k}\right\}$ form a basis of the subalgebra $\mathfrak{h}$. In our case $\left\{e_{n+1}, \ldots, e_{n+k}\right\}=\left\{h, y, e_{i}^{j}\right\}$. An arbitrary element $\varphi \in \operatorname{Hom}\left(\wedge^{2} \mathfrak{g}_{-}, \mathfrak{g}\right)$ defined by constants $C_{i j}^{k}$, where

$$
\varphi\left(e_{i}, e_{j}\right)=\sum_{k=1}^{n+k} C_{i j}^{k} e_{k}, \quad 1 \leq i, j \leq n .
$$

The structure function $C: P \rightarrow \operatorname{Hom}\left(\wedge^{2} \mathfrak{g}_{-}, \mathfrak{g}\right)$ defines functions $C_{i j}^{k}(p)$. If

$$
\omega=\sum \omega_{i} e_{i}, \quad \Omega=\sum \Omega^{k} e_{k},
$$

then the functions $C_{i j}^{k}(p)$ can be found from the decomposition of the curvature tensor $\Omega$ in terms of forms $\omega_{i}$ :

$$
\Omega^{k}=\sum C_{i j}^{k} \omega_{i} \wedge \omega_{j}
$$

Let $\Omega^{i}$ be one of the 2 -forms $\bar{\Omega}_{-3}^{i}, \bar{\Omega}_{-2}^{i}, \bar{\Omega}_{-1}^{i}, \bar{\Omega}_{x}, \bar{\Omega}_{h}, \bar{\Omega}_{j}^{i}$. We can write it explicitly as:

$$
\Omega^{i}=\sum_{p, q=1}^{3} \Omega^{i}\left[\bar{\omega}_{-q}^{j}, \bar{\omega}_{-p}^{k}\right] \bar{\omega}_{-q}^{j} \wedge \bar{\omega}_{-p}^{k}+\sum_{p=1}^{3} \Omega^{i}\left[\bar{\omega}_{x}, \bar{\omega}_{-p}^{k}\right] \bar{\omega}_{x} \wedge \bar{\omega}_{-p}^{k} .
$$

Then $\Omega^{i}\left[\bar{\omega}_{-q}^{j}, \bar{\omega}_{-p}^{k}\right]$ and $\Omega^{i}\left[\bar{\omega}_{x}, \bar{\omega}_{-p}^{k}\right]$ are the coefficients of the structure function of the Cartan connection $\omega$. The grading of Lie algebra $\mathfrak{g}$ induces degree of the coefficients $\Omega^{i}\left[\bar{\omega}_{-q}^{j}, \bar{\omega}_{-p}^{k}\right]$ and $\Omega^{i}\left[\bar{\omega}_{x}, \bar{\omega}_{-p}^{k}\right]$.

Definition 4. We say that Cartan connection associated with the equation (1) is characteristic if the following conditions on a curvature is satisfied:

- all coefficients of degree $\leq 1$ are equal to 0 ;

- in degree 2 we have $\bar{\Omega}_{h}\left[\bar{\omega}_{x} \wedge \bar{\omega}_{-1}^{i}\right]=0, \bar{\Omega}_{j}^{i}\left[\bar{\omega}_{x} \wedge \bar{\omega}_{-1}^{k}\right]=0, \bar{\Omega}_{x}\left[\bar{\omega}_{x} \wedge \bar{\omega}_{-2}^{i}\right]=0, \bar{\Omega}_{-1}^{i}\left[\bar{\omega}_{x} \wedge \bar{\omega}_{-2}^{i}\right]=0$; 
- in degree 3 we have $\bar{\Omega}_{y}\left[\bar{\omega}_{x} \wedge \bar{\omega}_{-1}^{i}\right]=0, \bar{\Omega}_{h}\left[\bar{\omega}_{x} \wedge \bar{\omega}_{-2}^{i}\right]=0, \bar{\Omega}_{j}^{i}\left[\bar{\omega}_{x} \wedge \bar{\omega}_{-2}^{k}\right]=0$;

- in degree 4 we have $\bar{\Omega}_{y}\left[\bar{\omega}_{x} \wedge \bar{\omega}_{-2}^{i}\right]=0$.

In other worlds these conditions define the subspace $U$ and Cartan connection is characteristic if and only if it belongs to $U$.

Theorem 1. There exists a unique characteristic Cartan connection associated with the equation (1).

Proof. We will proceed with parametric computations of characteristic Cartan connection in the forth section of the paper. We will fix a section $s: \mathcal{E} \rightarrow P$ and prove that locally for every equation there exists a unique Cartan connection $\omega$ with structure function pullback $s^{*} C: \mathcal{E} \rightarrow \operatorname{Hom}\left(\wedge^{2} \mathfrak{g}_{-}, \mathfrak{g}\right)$ takes values in the space $U$. Now we show that the characteristic Cartan connection is uniquely globally defined with this data.

Take a covering $U_{\alpha}$ of the space $E$ and construct a Cartan connection $\omega_{\alpha}$ on each trivial fibre bundle $\pi_{\alpha}: U_{\alpha} \times H \rightarrow U_{\alpha}$. Let $s_{\alpha}$ and $s_{\beta}$ be the trivial sections of the fibre bundles $\pi_{\alpha}$ and $\pi_{\beta}$. Let $\widetilde{\omega}_{\alpha}=s_{\alpha}^{*} \omega_{\alpha}$ and $\widetilde{\omega}_{\beta}=s_{\beta}^{*} \omega_{\beta}$. Since forms $\omega_{\alpha}$ and $\omega_{\beta}$ are uniquely defined there exists a unique function

$$
\varphi_{\alpha \beta}: U_{\alpha} \cap U_{\beta} \rightarrow H,
$$

such that

$$
\omega_{\beta}=\operatorname{Ad}\left(\varphi_{\alpha \beta}^{-1}\right) \omega_{\alpha}+\varphi_{\alpha \beta}^{*} \omega_{H},
$$

where $\omega_{H}$ is Maurer-Cartan form of the Lie group $H$. The functions $\varphi_{\alpha \beta}$ uniquely define a principle $H$-bundle with the Cartan connection $\omega$.

In order to prove that the structure function $C$ of the Cartan connection $\omega$ takes values in the space $U$ it is sufficient to show that $U$ is $\operatorname{Ad}(H)$-invariant.

Note that the action of $G_{0}$ preserves the zero condition on the structure function of the characteristic connection. We need only to check that the space $U$ is $\exp (y)$-invariant or equally $\operatorname{ad}(y)$ invariant. The action of the element $y$ has degree one. Conditions on the curvature of the Theorem 1 are ad(y)-invariant up degree 2, since all components of degree less than 2 are equal to zero. Finally, the conditions of degree 3 and 4 are $\operatorname{ad}(y)$-invariant, since the coefficients $\bar{\Omega}_{y}\left[\bar{\omega}_{x} \wedge \bar{\omega}_{-1}^{i}\right], \bar{\Omega}_{h}\left[\bar{\omega}_{x} \wedge \bar{\omega}_{-2}^{i}\right], \bar{\Omega}_{j}^{i}\left[\bar{\omega}_{x} \wedge \bar{\omega}_{-2}^{k}\right]$ and $\bar{\Omega}_{y}\left[\bar{\omega}_{x} \wedge \bar{\omega}_{-2}^{i}\right]$ can be obtained only from $\bar{\Omega}_{h}\left[\bar{\omega}_{x} \wedge \bar{\omega}_{-1}^{i}\right]$, $\bar{\Omega}_{x}\left[\bar{\omega}_{x} \wedge \bar{\omega}_{-2}^{i}\right], \bar{\Omega}_{j}^{i}\left[\bar{\omega}_{x} \wedge \bar{\omega}_{-1}^{k}\right], \bar{\Omega}_{h}\left[\bar{\omega}_{x} \wedge \bar{\omega}_{-2}^{i}\right]$ and $\bar{\Omega}_{y}\left[\bar{\omega}_{x} \wedge \bar{\omega}_{-1}^{i}\right]$ which all are zero for characteristic Cartan connection. This ends the proof of a global existence of the form $\omega$.

Let $V$ be an arbitrary finite-dimensional vector space and let $f$ be a smooth function $f: P \rightarrow V$. Denote by $L_{0}(f)$ the space of all functions of the form $\left\langle f, v^{*}\right\rangle$, where $v^{*} \in V^{*}$ and by $L(f)$ the algebra generated by elements from $L_{0}(f)$ and all their covariant derivatives. For example, the algebra $L(C)$, where $C$ is structure function of the Cartan connection $\omega$, consists of local invariants of the connection $\omega$.

Definition 5. We say that functions $f_{i}$ are the fundamental system of differential invariants for the structure with Cartan connection $\omega$ if $L\left(f_{i}\right)=L(C)$.

The key to calculation of the fundamental system of differential invariants is to determine which parts of the curvature are expressed through another. In [3] it is shown that fundamental invariants of holonomic differential equation lie in non-negative harmonic part of the curvature of the normal Cartan connection. In general we have approximately the same situation: there is one to one correspondence between fundamental differential invariants of the characteristic Cartan connection and $H_{+}^{2}\left(\mathfrak{g}_{-}, \mathfrak{g}\right)$ part of the structure function. Here $H_{+}^{2}\left(\mathfrak{g}_{-}, \mathfrak{g}\right)$ is the nonnegative part of the second Lie algebra cohomology group. 
Proposition 1. Let $\omega$ be a Cartan connection of type $(G, H)$ on a principal $H$-bundle $P$, where $(G, H)$ is an arbitrary pair of Lie group and its subgroup. Assume that the Lie algebra $\mathfrak{g}$ is a graded Lie algebra of the Lie group $G$ with the negative part $\mathfrak{g}_{-}$. Assume that a structure function of $\omega$ takes values in subspace $W \subset \operatorname{Hom}\left(\wedge^{2} \mathfrak{g}_{-}, \mathfrak{g}\right)$ and has only components of positive degree. Then a Ker $\partial \cap W$ part of the structure function forms a system of fundamental differential invariants.

Proof. The algebra of differential invariants is generated by the structure function coefficients. We will use the Bianchi identity to show that some coefficients of the characteristic Cartan connection curvature are obtained from the image of the operator $\partial$.

Let $e_{i}$ be the basis of the Lie algebra $\mathfrak{g}, X_{i}$ be the corresponding fundamental vector fields on $P$ and $\omega^{i}$ be the dual coframe. We can write the Cartan connection $\omega$ in the form:

$$
\omega=\omega^{i} e_{i} \text {. }
$$

Assume that the Lie algebra $\mathfrak{g}$ has structure constants $A_{i j}^{k}$. That means that:

$$
\left[e_{i}, e_{j}\right]=A_{i j}^{k} e_{k}
$$

Write the curvature of the Cartan connection $\omega$ in coordinates:

$$
\Omega=C_{i j}^{k} \omega^{i} \wedge \omega^{j} e_{k}
$$

Then the following equality is fulfilled:

$$
\mathrm{d} \omega^{k}=\left(C_{i j}^{k}-A_{i j}^{k}\right) \omega^{i} \wedge \omega^{j} .
$$

Now apply the Bianchi identity $\mathrm{d} \Omega=[\Omega, \omega]$ to the equation (2):

$$
\left(\frac{\partial C_{i j}^{k}}{\partial X_{l}} \omega_{l} \wedge \omega_{i} \wedge \omega_{j} e_{k}+C_{i j}^{k} \mathrm{~d} \omega_{i} \wedge \omega_{j} e_{k}+C_{i j}^{k} \omega_{i} \wedge \mathrm{d} \omega_{j} e_{k}\right)=C_{i j}^{k}\left[e_{k}, e_{l}\right] \omega_{i} \wedge \omega_{j} \wedge \omega_{l} .
$$

Express the covariant derivative of the structure function:

$$
\begin{aligned}
\frac{\partial C_{i j}^{p}}{\partial X_{l}} \omega_{l} \wedge \omega_{i} \wedge \omega_{j} e_{p} & =\left(-C_{k l}^{p} \mathrm{~d} \omega_{k} \wedge \omega_{l}-C_{k l}^{p} \omega_{k} \wedge \mathrm{d} \omega_{l}+C_{i j}^{k} A_{k l}^{p} \omega_{i} \wedge \omega_{j} \wedge \omega_{l}\right) e_{p} \\
& =C_{k l}^{p}\left(C_{i j}^{k}-A_{i j}^{k}\right) \omega_{i} \wedge \omega_{j} \wedge \omega_{l} e_{p}+C_{i j}^{k} A_{k l}^{p} \omega_{i} \wedge \omega_{j} \wedge \omega_{l} e_{p} .
\end{aligned}
$$

We get that:

$$
\begin{aligned}
& \frac{\partial C_{i j}^{p}}{\partial X_{l}} \omega_{l} \wedge \omega_{i} \wedge \omega_{j} e_{p}-C_{k l}^{p} C_{i j}^{k} \omega_{i} \wedge \omega_{j} \wedge \omega_{l} e_{p} \\
& \quad=C_{k l}^{p} A_{i j}^{k} \omega_{i} \wedge \omega_{j} \wedge \omega_{l} e_{p}+C_{i j}^{k} A_{k l}^{p} \omega_{i} \wedge \omega_{j} \wedge \omega_{l} e_{p} .
\end{aligned}
$$

If we take the $\operatorname{Hom}\left(\wedge^{3} \mathfrak{g}_{-}, \mathfrak{g}\right)$ part of (3) (i.e. assume that $\left.\omega_{l} \in \mathfrak{g}_{-}^{*}\right)$ we get that the right side of the (3) is exactly the Lie cohomology differential.

On the right side of (3) coefficients have the same degree as in the curvature. On the other hand coefficients on the left side have an increased degree. So, we have obtained that coefficients which are mapped to the im $\partial$ can be expressed through the covariant derivative of the coefficients of the lower degree. This proves the proposition.

Remark 1. Note that if intersection of $W$ and $\operatorname{Im} \partial$ is zero then subspace Ker $\partial \cap W$ is generated by representatives of $H_{+}^{2}\left(\mathfrak{g}_{-}, \mathfrak{g}\right)$. 
Theorem 2. The following invariants are fundamental differential invariants for the system of third order ODEs:

$$
\begin{aligned}
\left(W_{2}\right)_{j}^{i} & =\operatorname{tr}_{0}\left(\frac{\partial f^{i}}{\partial p^{j}}-\frac{\mathrm{d}}{\mathrm{dx}} \frac{\partial f^{i}}{\partial q^{j}}+\frac{1}{3} \frac{\partial f^{i}}{\partial q^{k}} \frac{\partial f^{k}}{\partial q^{j}}\right), \quad\left(I_{2}\right)_{j, k}^{i}=\operatorname{tr}_{0}\left(\frac{\partial^{2} f^{i}}{\partial q^{j} \partial q^{k}}\right), \\
\left(W_{3}\right)_{j}^{i} & =\frac{\partial f^{i}}{\partial y^{j}}+\frac{1}{3} \frac{\partial f^{i}}{\partial q^{k}} \frac{\partial f^{k}}{\partial p^{j}}-\frac{1}{2} \frac{d}{d x} \frac{\partial f^{i}}{\partial p^{j}}+\frac{1}{6} \frac{d^{2}}{d x^{2}} \frac{\partial f^{i}}{\partial q^{j}}-\frac{2}{27}\left(\frac{\partial f^{i}}{\partial q^{k}}\right)^{3}-\frac{1}{18} \frac{\partial f^{i}}{\partial q^{k}} \frac{d}{d x} \frac{\partial f^{k}}{\partial q^{j}} \\
& -\frac{5}{18} \frac{d}{d x}\left(\frac{\partial f^{i}}{\partial q^{k}}\right) \frac{\partial f^{k}}{\partial q^{j}} \\
\left(I_{4}\right)_{j, k} & =-\frac{\partial H_{k}^{-1}}{\partial p_{j}}+\frac{\partial}{\partial q_{j}} \frac{\partial}{\partial q_{k}} H^{x}-\frac{\partial}{\partial q_{k}} \frac{\mathrm{d}}{\mathrm{dx}} H_{j}^{-1}-\frac{\partial}{\partial q^{k}}\left(H_{l}^{-1} \frac{\partial f^{l}}{\partial q^{j}}\right)+2 H_{j}^{-1} H_{k}^{-1}
\end{aligned}
$$

where

$$
H_{j}^{-1}=\frac{1}{6(m+1)}\left(\frac{\partial^{2} f^{i}}{\partial q^{i} \partial q^{j}}\right) \quad \text { and } \quad H^{x}=-\frac{1}{4 m}\left(\frac{\partial f^{i}}{\partial p^{i}}-\frac{\mathrm{d}}{\mathrm{dx}} \frac{\partial f^{i}}{\partial q^{i}}+\frac{1}{3} \frac{\partial f^{i}}{\partial q^{k}} \frac{\partial f^{k}}{\partial q^{i}}\right)
$$

Proof. We will use Proposition 1. The fundamental differential invariants is in one to one correspondence with the cohomology group $H_{+}^{2}\left(\mathfrak{g}_{-}, \mathfrak{g}\right)$. For the case of the system of ODEs of the third order the Lie cohomology group $H_{+}^{2}\left(\mathfrak{g}_{-}, \mathfrak{g}\right)$ was studied in [6]. The main result of that work is that the space $H_{+}^{2}\left(\mathfrak{g}_{-}, \mathfrak{g}\right)$ has the following decomposition:

\begin{tabular}{|c|c|}
\hline Degree & Space \\
\hline-1 & $v_{6}^{0} \otimes \wedge^{2}\left(W^{*}\right) \otimes W$ \\
0 & $v_{4}^{0} \otimes S_{0}^{2}\left(W^{*}\right) \otimes W$ \\
0 & $v_{4}^{0} \otimes \wedge^{2}\left(W^{*}\right) \otimes W$ \\
1 & $v_{2}^{0} \otimes \wedge^{2} W^{*} \otimes W / V_{2} \otimes W^{*}$ \\
2 & $x^{*} \otimes \mathbb{R} y \otimes \mathfrak{s l}(W)$ \\
2 & $v_{0}^{0} \otimes S^{2}\left(W^{*}\right) \otimes W$ \\
3 & $x^{*} \otimes \mathbb{R} y^{2} \otimes \mathfrak{g l}(W)$ \\
4 & $v_{0}^{0} \otimes S^{2}\left(W^{*}\right)$ \\
3 & $v_{2}^{0}$ if $m=2$ \\
\hline
\end{tabular}

Here $v_{k}^{0}$ is the lowest vector of corresponding $(k+1)$-dimensional $\mathfrak{s} l_{2}$-module $V_{k}$.

Now we list the result table with the corresponding invariant. We start from degree 2 since all part of curvature of degree less than 2 is zero.

\begin{tabular}{|c|c|c|c|}
\hline Degree & Space & Part of the curvature & Invariant \\
\hline 2 & $x^{*} \otimes \mathbb{R} y \otimes \mathfrak{s l}(W)$ & $\Omega_{-1}^{i}\left[\omega_{x} \wedge \omega_{-2}^{j}\right]$ & $W_{2}$ \\
2 & $v_{0}^{0} \otimes S^{2}\left(W^{*}\right) \otimes W$ & $\Omega_{-2}^{i}\left[\omega_{-1}^{j} \wedge \omega_{-3}^{k}\right]$ & $I_{2}$ \\
3 & $x^{*} \otimes \mathbb{R} y^{2} \otimes \mathfrak{g l}(W)$ & $\Omega_{-1}^{i}\left[\omega_{x} \wedge \omega_{-3}^{j}\right]$ & $W_{3}$ \\
4 & $v_{0}^{0} \otimes S^{2}\left(W^{*}\right)$ & $\Omega_{y}\left[\omega_{x} \wedge \omega_{-3}^{j}\right]$ & $I_{4}$ \\
3 & $v_{2}^{0}$ if $m=2$ & $\Omega_{y}\left[\omega_{-1}^{2} \wedge \omega_{-2}^{1}\right]$ & $\equiv 0$ \\
\hline
\end{tabular}

Corollary 1. The system (1) is equivalent to the trivial one via point transformations if and only if all invariants $I_{2}, W_{2}, W_{3}, I_{4}$ vanish identically.

Example 1 (Differential equations on circles in $\mathbb{R}^{n}$ ). As application of the previous results we compute invariants of the system of third order ODEs on circles in Euclidean space. 
Lemma 1. Let $E$ be the $(m+1)$-dimensional Euclidean space with the orthonormal basis $\left\{e_{0}, \ldots, e_{n}\right\}$ and the coordinates $\left\{r_{0}, r_{1}, \ldots, r_{n}\right\}$. Then the equation of circles in $E$ parametrized by the coordinate $r_{0}$ is:

$$
\dddot{r}_{i}=3 \ddot{r}_{i} \frac{\sum_{j=1}^{m} \dot{r}_{j} \ddot{r}_{j}}{1+\sum_{j=1}^{m} \dot{r}_{j}^{2}}, \quad i=1, \ldots, m
$$

This equation is invariant under conformal transformations of E.

Proof. Let the curve $R(t)=\left(r_{0}(t), \ldots, r_{n}(t)\right)$ be a circle. Assume now that $r_{0}(t)=t$. We have

$$
\dddot{R}(t)=a(t) \ddot{R}(t)+b(t) \dot{R}(t),
$$

since $R(t)$ is 2 -dimensional curve. Next, $b(t)=0$ in our parametrization, since

$$
0=\dddot{r}_{0}(t)=a(t) \ddot{r}_{0}(t)+b(t) \dot{r}_{0}(t)=b(t) .
$$

To determine $a(t)$ note that

$$
(R(t)-C, R(t)-C)=d
$$

for some constant $d$ and $C \in E$. Differentiating, we get:

$$
\begin{aligned}
& (\dot{R}(t), R(t)-C)=0, \\
& (\ddot{R}(t), R(t)-C)=-(\dot{R}(t), \dot{R(} t)), \\
& (\dddot{R}(t), R(t)-C)+3(\ddot{R}(t), \dot{R}(t))=0 .
\end{aligned}
$$

Now substitute (4) into previous formula:

$$
\begin{aligned}
& (a(t) \ddot{R}(t)+b(t) \dot{R}(t), R(t)-C)=-3(\ddot{R}(t), \dot{R}(t)), \\
& (a(T) \ddot{R}(t), R(t)-C)=-a(t)(\dot{R}(t), \dot{R}(t))=-3(\ddot{R}(t), \dot{R}(t)) .
\end{aligned}
$$

We get that

$$
a(t)=3 \frac{(\ddot{R}(t), \dot{R}(t))}{(\dot{R}(t), \dot{R}(t))} .
$$

Substituting $a(t)$ into (4) we get our equations.

Proposition 2. For differential equation on conformal circles invariants $W_{2}, I_{2}, W_{3}$ vanish identically. Invariant $I_{4}$ has the following form:

$$
\left(I_{4}\right)_{j}^{i}=\frac{1}{2} \delta_{j}^{i} \frac{1}{1+\sum_{k=1}^{m} \dot{r}_{k}^{2}}-\frac{1}{2} \frac{\dot{r}_{i} \dot{r}_{j}}{\left(1+\sum_{k=1}^{m} \dot{r}_{k}^{2}\right)^{2}} .
$$

Proof. The proof is straightforward applying of the formulas from Theorem 2.

Remark 2. There are other equations satisfying $W_{2}=I_{2}=W_{3}=0$. For example, it is an union of a system on circles in $R^{n-k}$ and a system of $k$ trivial equations. It would be interesting to characterize geometrically the class of such equations. 


\section{Parametric computation of the characteristic Cartan connection}

Consider a system of third-order ordinary differential equations of the form

$$
\left(y^{i}\right)^{\prime \prime \prime}=f^{i}\left(x, y^{j},\left(y^{k}\right)^{\prime},\left(y^{l}\right)^{\prime \prime}\right),
$$

where $i, j=1, \ldots, m$ with $m \geq 2$. It determines a holonomic differential equation $\mathcal{E} \subset$ $J^{3}\left(\mathbb{R}^{m+1}, 1\right)$. Let us use the following coordinate system on the equation $\mathcal{E}$ :

$$
x, y_{1}, \ldots, y_{m}, p_{1}=y_{1}^{\prime}, \ldots, p_{m}=y_{m}^{\prime}, q_{1}=y_{1}^{\prime \prime}, \ldots, q_{m}=y_{m}^{\prime \prime} .
$$

We choose a coframe $\theta$ on the surface $\mathcal{E}$ :

$$
\begin{aligned}
& \theta_{x}=d x \\
& \theta_{-1}^{i}=d q^{i}-f^{i}(x, y, p, q) d x, \quad i=1, \ldots, m ; \\
& \theta_{-2}^{i}=d p^{i}-q^{i} d x, \quad i=1, \ldots, m ; \\
& \theta_{-3}^{i}=d y^{i}-p^{i} d x, \quad i=1, \ldots, m .
\end{aligned}
$$

To connect our computation on the surface $\mathcal{E}$ with the principle bundle $P$ let us use the following uniquely defined section $s: \mathcal{E} \rightarrow P$ with relations:

$$
\begin{aligned}
& s^{*} \bar{\omega}_{-3}^{i}=\theta_{-3}^{i}, \\
& s^{*} \bar{\omega}_{h} \equiv 0 \quad \bmod \left\langle\theta_{-3}^{i}, \theta_{-2}^{i}, \theta_{-1}^{i}\right\rangle, \\
& s^{*} \bar{\omega}_{x} \equiv-\theta_{x} \quad \bmod \left\langle\theta_{-3}^{i}, \theta_{-2}^{i}, \theta_{-1}^{i}\right\rangle .
\end{aligned}
$$

Define a pullback $\omega: T \mathcal{E} \rightarrow \mathfrak{g}$ by the formula $\omega=s^{*} \bar{\omega}$. Let $\bar{\Omega}$ be a curvature tensor of $\bar{\omega}$, and let $\Omega=s^{*} \bar{\Omega}$. We see that

$$
\begin{aligned}
\Omega= & \Omega_{-3}^{i} v_{0} \otimes e_{i}+\Omega_{-2}^{i} v_{1} \otimes e_{i}+\Omega_{-1}^{i} v_{2} \otimes e_{i}+\Omega_{x} x+\Omega_{h} h+\Omega_{i}^{j} e_{j}^{i}+\Omega_{y} y \\
= & \left(d \omega_{-3}^{i}+\omega_{x} \wedge \omega_{-2}^{i}+2 \omega_{h} \wedge \omega_{-3}^{i}+\omega_{j}^{i} \wedge \omega_{-3}^{j}\right) v_{0} \otimes e_{i} \\
& +\left(d \omega_{-2}^{i}+\omega_{x} \wedge \omega_{-1}^{i}+\omega_{j}^{i} \wedge \omega_{-2}^{j}+2 \omega_{y} \wedge \omega_{-3}^{i}\right) v_{1} \otimes e_{i} \\
& +\left(d \omega_{-1}^{i}-2 \omega_{h} \wedge \omega_{-1}^{i}+\omega_{j}^{i} \wedge \omega_{-1}^{j}+2 \omega_{y} \wedge \omega_{-2}^{i}\right) v_{2} \otimes e_{i} \\
& +\left(d \omega_{x}+2 \omega_{h} \wedge \omega_{x}\right) x+\left(d \omega_{h}+\omega_{x} \wedge \omega_{y}\right) h+\left(d \omega_{j}^{i}+\omega_{k}^{i} \wedge \omega_{j}^{k}\right) e_{i}^{j}+\left(d \omega_{y}-2 \omega_{h} \wedge \omega_{y}\right) y .
\end{aligned}
$$

An arbitrary Cartan connection adapted to equation (1) has the form:

$$
\begin{aligned}
& \omega_{-3}^{i}=\theta_{-3}^{i}, \\
& \omega_{-2}^{i}=\alpha_{j}^{i} \theta_{-2}^{j}+A_{j}^{i} \theta_{-3}^{j}, \\
& \omega_{-1}^{i}=\beta_{j}^{i} \theta_{-1}^{j}+B_{j}^{i} \theta_{-2}^{j}+C_{j}^{i} \theta_{-3}^{j}, \\
& \omega_{x}=-\theta_{x}+D_{j} \theta_{-2}^{j}+E_{j} \theta_{-3}^{j}, \\
& \omega_{h}=F_{j}^{-1} \theta_{-1}^{j}+F_{j}^{-2} \theta_{-2}^{j}+F_{j}^{-3} \theta_{-3}^{j}, \\
& \omega_{j}^{i}=G_{j}^{i, x} \theta_{x}+G_{j k}^{i,-1} \theta_{-1}^{k}+G_{j k}^{i,-2} \theta_{-2}^{k}+G_{j k}^{i,-3} \theta_{-3}^{k}, \\
& \omega_{y}=H^{x} \theta_{x}+H_{j}^{-1} \theta_{-1}^{j}+H_{j}^{-2} \theta_{-2}^{j}+H_{j}^{-3} \theta_{-3}^{j} .
\end{aligned}
$$

In degree 0 of the curvature we have two nonzero components:

$$
\Omega_{-3}^{i} \bmod \left\langle\theta_{-2} \wedge \theta_{-2}, \theta_{-3}\right\rangle=\theta^{x} \wedge \theta_{-2}^{i}-\alpha_{j}^{i} \theta^{x} \wedge \theta_{-2}^{j},
$$




$$
\Omega_{-2}^{i} \bmod \left\langle\theta_{-2}, \theta_{-3}\right\rangle=\theta^{x} \wedge \theta_{-1}^{i}-\beta_{j}^{i} \theta^{x} \wedge \theta_{-1}^{j} .
$$

Assume these two equalities is zero and get $\alpha_{j}^{i}=\delta_{j}^{i}$ and $\beta_{j}^{i}=\delta_{j}^{i}$.

We have three nonzero components in degree 1 . The first component is:

$$
\begin{aligned}
\Omega_{-3}^{i} & \bmod \left\langle\theta_{-2} \wedge \theta_{-3}, \theta_{-3} \wedge \theta_{-3}\right\rangle \\
& =-\theta_{x} \wedge A_{j}^{i} \theta_{-2}^{j}+D_{j} \theta_{-2}^{j} \wedge \theta_{-2}^{i}+G_{i}^{i, x} \theta_{x} \wedge \theta_{-3}^{j}+G_{j k}^{i,-1} \theta_{-1}^{k} \wedge \theta_{-3}^{j}+2 F_{j}^{-1} \theta_{-1}^{j} \wedge \theta_{-3}^{i} .
\end{aligned}
$$

The second component is:

$$
\begin{aligned}
\Omega_{-2}^{i} & \bmod \left\langle\theta_{-2} \wedge \theta_{-2}, \theta_{-3}\right\rangle \\
& =A_{j}^{i} \theta_{x} \wedge \theta_{-2}^{j}+D_{j} \theta_{-2}^{j} \wedge \theta_{-1}^{i}-\theta_{x} \wedge B_{j}^{i} \theta_{-2}^{j}+G_{j}^{i, x} \theta_{x} \wedge \theta_{-2}^{j}+G_{j k}^{i,-1} \theta_{-1}^{k} \wedge \theta_{-2}^{j} .
\end{aligned}
$$

The third component is:

$$
\begin{aligned}
\Omega_{-1}^{i} & \bmod \left\langle\theta_{-2}, \theta_{-3}\right\rangle \\
= & \frac{\partial f^{i}}{\partial q^{j}} \theta_{x} \wedge \theta_{-1}^{j}+B_{j}^{i} \theta_{x} \wedge \theta_{-1}^{j}-2 F_{j}^{-1} \theta_{-1}^{j} \wedge \theta_{-1}^{i}+G_{j}^{i, x} \theta_{x} \wedge \theta_{-1}^{j}+G_{j k}^{i,-1} \theta_{-1}^{k} \wedge \theta_{-1}^{j} .
\end{aligned}
$$

After applying zero conditions to these parts of the curvature we obtain

$$
A_{j}^{i}=G_{j}^{i, x}=\frac{1}{2} B_{j}^{i}=-\frac{1}{3} \frac{\partial f^{i}}{\partial q^{j}}, \quad D_{j}=F_{j}^{-1}=G_{j k}^{i,-1}=0 .
$$

Proceed now to the second degree

$$
\begin{aligned}
\Omega_{-1}^{i} & \bmod \left\langle\theta_{-2} \wedge \theta_{-2}, \theta_{-3}\right\rangle \\
= & \frac{\partial f^{i}}{\partial p_{j}} \theta_{x} \wedge \theta_{-2}^{j}+2 \frac{d A_{j}^{i}}{d x} \theta_{x} \wedge \theta_{-2}^{j}+2 \frac{\partial A_{j}^{i}}{\partial q_{k}} \theta_{-1}^{k} \wedge \theta_{-2}^{j}+C_{j}^{i} \theta_{x} \wedge \theta_{-2}^{j}-2 F_{j}^{-2} \theta_{-2}^{j} \wedge \theta_{-1}^{i} \\
& +G_{j k}^{i,-2} \theta_{-2}^{k} \wedge \theta_{-1}^{j}+2 H^{x} \theta_{x} \wedge \theta_{-2}^{i}+2 H_{j}^{-1} \theta_{-1}^{j} \wedge \theta_{-2}^{i}+G_{k}^{i, x} \theta_{x} \wedge B_{j}^{k} \theta_{-2}^{j} .
\end{aligned}
$$

We have:

$$
\Omega_{-1}^{i}\left[\theta_{x} \wedge \theta_{-2}^{j}\right]=\frac{\partial f^{i}}{\partial p_{j}}+2 \frac{d A_{j}^{i}}{d x}+C_{j}^{i}+2 H^{x}+2 A_{k}^{i} A_{j}^{k} .
$$

Assuming the previous tensor is zero, we obtain:

$$
C_{j}^{i}=-\left(\frac{\partial f^{i}}{\partial p_{j}}+2 \frac{d A_{j}^{i}}{d x}+2 H^{x}+2 A_{k}^{i} A_{j}^{k}\right) .
$$

Next curvature component contains all second order invariants:

$$
\begin{aligned}
\Omega_{-2}^{i} & \bmod \left\langle\theta_{-2} \wedge \theta_{-3}, \theta_{-3} \wedge \theta_{-3}\right\rangle \\
= & \frac{d A_{j}^{i}}{d x} \theta_{x} \wedge \theta_{-3}^{j}+\frac{\partial A_{j}^{i}}{\partial q_{k}} \theta_{-1}^{k} \wedge \theta_{-3}^{j}-\theta_{x} \wedge C_{j}^{i} \theta_{-3}^{j}+E_{j} \theta_{-3}^{j} \wedge \theta_{-1}^{i}+G_{j}^{i, x} \theta_{x} \wedge A_{k}^{j} \theta_{-3}^{k} \\
& +2 H^{x} \theta_{x} \wedge \theta_{-3}^{i}+2 H_{j}^{-1} \theta_{-1}^{j} \wedge \theta_{-3}^{i}+G_{j k}^{i,-2} \theta_{-2}^{k} \wedge \theta_{-2}^{j}+G_{j k}^{i,-1} \theta_{k}^{-1} \wedge A_{l}^{j} \theta_{-3}^{l} .
\end{aligned}
$$

In coefficient $\Omega_{-2}^{i}\left[\theta_{-1}^{k} \wedge \theta_{-3}^{j}\right]$ we get invariant $I_{2}$

$$
\Omega_{-2}^{i}\left[\theta_{-1}^{k} \wedge \theta_{-3}^{j}\right]=\frac{\partial A_{j}^{i}}{\partial q_{k}}-E_{j} \delta_{k}^{i}+2 H_{k}^{-1} \delta_{j}^{i}=\frac{\partial A_{j}^{i}}{\partial q_{k}}+2 H_{k}^{-1} \delta_{j}^{i}+2 F_{j}^{-2} \delta_{k}^{i} .
$$


Explicitly, the invariant $I_{2}$ is the following:

$$
I_{2}=\operatorname{tr}_{0}\left(\frac{\partial^{2} f^{i}}{\partial q_{j} \partial q_{k}}\right)
$$

where $\operatorname{tr}_{0}$ is a traceless part of the tensor.

In the coefficient

$$
\Omega_{-2}^{i}\left[\theta_{x} \wedge \theta_{-3}^{j}\right]=-C_{j}^{i} \frac{d A_{j}^{i}}{d x}+A_{k}^{i} A_{j}^{k}+2 H^{k} \delta_{j}^{i}
$$

we obtain a so-called generalized Wilczynski invariant. As shown in [2], a part of differential invariants of systems of ODEs comes from its linearisation. As in [2], we call them generalized Wilczynski invariants. In our case we have two Wilczynski invariants of degree 2 and 3. We denote them as $W_{2}$ and $W_{3}$ respectively. The second degree generalized Wilczynski invariant is the following:

$$
W_{2}=\operatorname{tr}_{0}\left(\frac{\partial f^{i}}{\partial p_{j}}-\frac{d}{d x} \frac{\partial f^{i}}{\partial q_{j}}+\frac{1}{3} \frac{\partial f^{i}}{\partial q_{k}} \frac{\partial f^{k}}{\partial q_{j}}\right) .
$$

Normalizing the trace of previous tensor to zero we obtain:

$$
H^{x}=-\frac{1}{4 m}\left(\frac{\partial f^{i}}{\partial p_{i}}+3 \frac{d A_{i}^{i}}{d x}+3 A_{k}^{i} A_{i}^{k}\right) .
$$

It remains to compute only $\mathfrak{s l}_{2} \times \mathfrak{g l}_{m}$ part of the curvature in degree 2 .

$$
\Omega_{x} \bmod \left\langle\theta_{-2} \wedge \theta_{-2}, \theta_{-3}\right\rangle=E_{j} \theta_{x} \wedge \theta_{-2}^{j}+2 F_{j} \theta_{x} \wedge \theta_{-2}^{j} .
$$

Assuming that it vanishes identically we get the following condition:

$$
E_{j}=-2 F_{j}^{-2}
$$

We have:

$$
\Omega_{h} \bmod \left\langle\theta_{-2}, \theta_{-3}\right\rangle=F_{j}^{-2} \theta_{x} \wedge \theta_{-1}^{j}-\theta_{x} \wedge \theta_{-1}^{j} H_{j}^{-1} .
$$

The condition $\Omega_{h}^{i}\left[\theta_{x} \wedge \theta_{-1}^{i}\right]=0$ gives equality $F_{j}^{-2}=H_{j}^{-1}$.

Assuming the trace of the tensor $\Omega_{-2}^{i}\left[\theta_{-1}^{j} \wedge \theta_{-3}^{k}\right]$ is equal to zero we get:

$$
F_{k}^{-2}=H_{k}^{-1}=-\frac{1}{2(m+1)} \frac{\partial A_{i}^{i}}{\partial q_{k}}
$$

The last part of degree 2 calculation is:

$$
\Omega_{j}^{i} \bmod \left\langle\theta_{-2}, \theta_{-3}\right\rangle=\frac{\partial A_{j}^{i}}{\partial q_{k}} \theta_{x} \wedge \theta_{k}^{-1}+G_{j k}^{i,-2} \theta_{x} \wedge \theta_{k}^{-1} .
$$

We obtain $G_{j k}^{i,-2}=\frac{\partial A_{j}^{i}}{\partial q_{k}}$ from condition $\bar{\Omega}_{j}^{i}\left[\bar{\omega}_{x} \wedge \bar{\omega}_{-1}^{k}\right]=0$.

Proceed now to the degree 3 . The first part of degree 3 we need to compute is $\Omega_{-1}^{i}$ :

$$
\begin{aligned}
\Omega_{-1}^{i} & \bmod \left\langle\theta_{-2} \wedge \theta_{-3}, \theta_{-3} \wedge \theta_{-3}\right\rangle=\frac{\partial f^{i}}{\partial y_{i}} \theta_{x} \wedge \theta_{-3}^{j}+\frac{\partial B_{j}^{i}}{\partial p_{k}} \theta_{-2}^{k} \wedge \theta_{-2}^{j} \\
+ & \frac{\partial C_{j}^{i}}{\partial x} \theta_{x} \wedge \theta_{-3}^{j}+\frac{\partial C_{j}^{i}}{\partial q_{k}} \theta_{-1}^{k} \wedge \theta_{-3}^{j}-2 F_{j}^{-3} \theta_{-3}^{j} \wedge \theta_{-1}^{i}-2 F_{j}^{-2} \theta_{-2}^{j} \wedge B_{k}^{i} \theta_{-2}^{k}
\end{aligned}
$$




$$
+G_{j k}^{i,-3} \theta_{-3}^{k}+G_{j k}^{i,-2} \theta_{-2}^{k} \wedge B_{j}^{i} \theta_{-2}^{j}+G_{j}^{i, x} \theta_{x} \wedge C_{k}^{j} \theta_{-3}^{k}+2 H_{j}^{-2} \theta_{-2}^{j} \wedge \theta_{-2}^{i}
$$

Wilczynski invariant $W_{3}$ appears as the $\Omega_{-1}^{i}\left[\theta_{x} \wedge \theta_{-3}^{j}\right]$ coefficient:

$$
\frac{\partial f^{i}}{\partial y_{j}}+\frac{d C_{j}^{i}}{d x}+A_{k}^{i} C_{j}^{k}+2 H^{x} A_{j}^{i}
$$

Direct computation shows that:

$$
\begin{aligned}
\Omega_{-1}^{i}\left[\theta_{x} \wedge \theta_{-3}^{j}\right]= & \frac{\partial f^{i}}{\partial y^{j}}+\frac{1}{3} \frac{\partial f^{i}}{\partial q^{k}} \frac{\partial f^{k}}{\partial p^{j}}-\frac{d}{d x} \frac{\partial f^{i}}{\partial p^{j}}+\frac{2}{3} \frac{d^{2}}{d x^{2}} \frac{\partial f^{i}}{\partial q^{j}}-\frac{2}{27}\left(\frac{\partial f^{i}}{\partial q^{j}}\right)^{3} \\
& -\frac{4}{9} \frac{\partial f^{i}}{\partial q^{k}} \frac{d}{d x} \frac{\partial f^{k}}{\partial q^{j}}-\frac{2}{9} \frac{d}{d x}\left(\frac{\partial f^{i}}{\partial q^{k}}\right) \frac{\partial f^{k}}{\partial q^{j}}-2 \delta_{j}^{i} H^{x} .
\end{aligned}
$$

Denote invariant $\Omega_{-1}^{i}\left[\theta_{x} \wedge \theta_{-3}^{j}\right]+\frac{1}{2} \frac{d}{d x} W_{2}$ as $W_{3}$. Invariant $W_{3}$ is equivalent to the fundamental invariant $\Omega_{-1}^{i}\left[\theta_{x} \wedge \theta_{-3}^{j}\right]$. It means that after replacing $\Omega_{-1}^{i}\left[\theta_{x} \wedge \theta_{-3}^{j}\right]$ with $W_{3}$ the system would remain fundamental. Explicitly the Wilczynski invariant $W_{3}$ is:

$$
\begin{aligned}
W_{3}= & \frac{\partial f^{i}}{\partial y^{j}}+\frac{1}{3} \frac{\partial f^{i}}{\partial q^{k}} \frac{\partial f^{k}}{\partial p^{j}}-\frac{1}{2} \frac{d}{d x} \frac{\partial f^{i}}{\partial p^{j}}+\frac{1}{6} \frac{d^{2}}{d x^{2}} \frac{\partial f^{i}}{\partial q^{j}} \\
& -\frac{2}{27}\left(\frac{\partial f^{i}}{\partial q^{j}}\right)^{3}-\frac{1}{18} \frac{\partial f^{i}}{\partial q^{k}} \frac{d}{d x} \frac{\partial f^{k}}{\partial q^{j}}-\frac{5}{18} \frac{d}{d x}\left(\frac{\partial f^{i}}{\partial q^{k}}\right) \frac{\partial f^{k}}{\partial q^{j}} .
\end{aligned}
$$

An expression $\left(\frac{\partial f^{i}}{\partial q^{j}}\right)^{3}$ here is the third power of the matrix $\frac{\partial f^{i}}{\partial q^{j}}$. Note that invariant $W_{3}$ has known analogue in the case of one differential equation of third order:

$$
\frac{\partial f}{\partial y}+\frac{1}{3} \frac{\partial f}{\partial q} \frac{\partial f}{\partial p}-\frac{1}{2} \frac{d}{d x} \frac{\partial f}{\partial p}+\frac{1}{6} \frac{d^{2}}{d x^{2}} \frac{\partial f}{\partial q}-\frac{2}{27}\left(\frac{\partial f}{\partial q}\right)^{3}-\frac{1}{3} \frac{\partial f}{\partial q} \frac{d}{d x} \frac{\partial f^{k}}{\partial q^{j}}
$$

The reader can find this invariant for example in Chern work [1]; also see Sato and Yoshikawa [8].

Let us compute the third degree normalization conditions.

$$
\begin{aligned}
\Omega_{h} & \bmod \left\langle\theta_{-2} \wedge \theta_{-2}, \theta_{-3}\right\rangle \\
& =F_{j}^{-3} \theta_{x} \wedge \theta_{-2}^{j}+\frac{d F_{j}^{-2}}{d x} \theta_{x} \wedge \theta_{-2}^{j}+\frac{\partial F_{j}^{-2}}{\partial q_{k}} \theta_{-1}^{k} \wedge \theta_{-2}^{j}-\theta_{x} \wedge A_{j}^{-2} \theta_{-2}^{j} .
\end{aligned}
$$

Thus:

$$
\Omega_{h}\left[\theta_{x} \wedge \theta_{-2}^{j}\right]=-H_{j}^{-2}+F_{j}^{-3}+\frac{d F_{j}^{-2}}{d x} .
$$

Normalizing this coefficient to 0 we obtain:

$$
F_{j}^{-3}=H_{j}^{-2}-\frac{d F_{j}^{-2}}{d x} .
$$

Next,

$$
\begin{aligned}
& \Omega_{j}^{i} \quad \bmod \left\langle\theta_{-2} \wedge \theta_{-2}, \theta_{-3}\right\rangle=\frac{\partial A_{j}^{i}}{\partial p_{k}} \theta_{-2}^{k} \wedge \theta_{x}+\frac{d G_{j k}^{i,-2}}{d x} \theta_{x} \wedge \theta_{-2}^{k}+G_{j k}^{i,-3} \theta_{x} \wedge \theta_{-2}^{k} \\
& +G_{k}^{i, x} \theta_{x} \wedge G_{j l}^{k,-2} \theta_{-2}^{l}+G_{k l}^{i,-2} \theta_{-2}^{k} \wedge G_{j}^{l, x} \theta_{x} .
\end{aligned}
$$


We have:

$$
\Omega_{j}^{i}\left[\theta_{x} \wedge \theta_{-2}^{k}\right]=-\frac{\partial A_{j}^{i}}{\partial p_{k}}+\frac{d G_{j k}^{i,-2}}{d x}+G_{j k}^{i,-3}+G_{l}^{i, x} G_{j k}^{l,-2}-G_{l k}^{i,-2} G_{j}^{l, x} .
$$

Assuming this coefficient is equal to 0 we get:

$$
G_{j k}^{i,-3}=\frac{\partial A_{j}^{i}}{\partial p_{k}}-\frac{d G_{j k}^{i,-2}}{d x}-G_{l}^{i, x} G_{j k}^{l,-2}+G_{l k}^{i,-2} G_{j}^{l x} .
$$

Finally,

$$
\begin{aligned}
\Omega_{y} & \bmod \left\langle\theta_{-2}, \theta_{-3}\right\rangle=\frac{\partial H^{x}}{\partial q_{i}} \theta_{-1}^{j} \wedge \theta_{x}+\frac{d H_{j}^{-1}}{d x} \theta_{x} \wedge \theta_{-1}^{j} \\
+ & \frac{\partial H_{j}^{-1}}{\partial q_{k}} \theta_{-1}^{k} \wedge \theta_{-1}^{j}+H_{j}^{-1} \frac{\partial f^{j}}{\partial q_{k}} \theta_{x} \wedge \theta_{-1}^{k}+H_{j}^{-2} \theta_{x} \wedge \theta_{-1}^{j} .
\end{aligned}
$$

The coefficient $\Omega_{y}\left[\theta_{-1}^{j} \wedge \theta_{x}\right]$ is the following:

$$
\frac{\partial H^{x}}{\partial q_{j}}-\frac{d H_{j}^{-1}}{d x}-H_{k}^{-1} \frac{\partial f^{k}}{\partial q_{j}}-H_{j}^{-2}
$$

Normalizing it to 0 we obtain:

$$
H_{j}^{-2}=\frac{\partial H^{x}}{\partial q_{j}}-\frac{d H_{j}^{-1}}{d x}-H_{k}^{-1} \frac{\partial f^{k}}{\partial q_{k}} .
$$

The last coefficient we need in degree 3 is $\Omega_{y}\left[\theta_{-1}^{k} \wedge \theta_{-1}^{j}\right]$ :

$$
\frac{\partial H_{j}^{-1}}{\partial q_{k}}-\frac{\partial H_{k}^{-1}}{\partial q_{j}}=0
$$

In the degree 4 we need to compute only one coefficient of curvature:

$$
\begin{aligned}
& \Omega_{y} \bmod \left\langle\theta_{-2} \wedge \theta_{-2}, \theta_{-3}\right\rangle=\frac{\partial H^{x}}{\partial p_{j}} \theta_{-2}^{j} \wedge \theta_{x}+\frac{\partial H_{j}^{-1}}{\partial p_{k}} \theta_{-2}^{k} \wedge \theta_{-1}^{j}+\frac{d H_{j}^{-2}}{d x} \theta_{x} \wedge \theta_{-2}^{j} \\
& +\frac{\partial H_{j}^{-2}}{\partial q_{k}} \theta_{k}^{-1} \wedge \theta_{-2}^{j}+H_{j}^{-1} \frac{\partial f^{j}}{\partial p_{k}} \theta_{x} \wedge \theta_{-2}^{k}+H_{j}^{-3} \theta_{x} \wedge \theta_{-2}^{j}-2 F_{j}^{-2} \theta_{-2}^{j} \wedge\left(H^{x} \theta_{x}+H_{k}^{-1} \theta_{-1}^{k}\right) .
\end{aligned}
$$

The Cartan connection coefficient $\Omega_{y}\left[\theta_{x} \wedge \theta_{-2}^{j}\right]$ has the following form:

$$
-\frac{\partial H^{x}}{\partial p_{j}}+\frac{d H_{j}^{-2}}{d x}+H_{k}^{-1} \frac{\partial f^{k}}{\partial q_{j}}-H_{j}^{-3} .
$$

Assuming it is equal to 0 we get:

$$
H_{j}^{-3}=-\frac{\partial H^{x}}{\partial p_{j}}+\frac{d H_{j}^{-2}}{d x}+H_{k}^{-1} \frac{\partial f^{k}}{\partial q_{j}} .
$$

Finally, invariant $I_{4}$ is the tensor $\Omega_{y}\left[\theta_{-1}^{k} \wedge \theta_{-2}^{j}\right]$ :

$$
-\frac{\partial H_{k}^{-1}}{\partial p_{j}}+\frac{\partial H_{j}^{-2}}{\partial q^{k}}+2 H_{j}^{-1} H_{k}^{-1}
$$




\section{References}

[1] Chern S.-S., The geometry of the differential equation $y^{\prime \prime \prime}=F\left(x, y, y^{\prime}, y^{\prime \prime}\right)$, Sci. Rep. Nat. Tsing Hua Univ. (A) 4 (1940), 97-111.

[2] Doubrov B., Contact trivialization of ordinary differential equations, in Differential Geometry and Its Applications (Opava, 2001), Math. Publ., Vol. 3, Silesian Univ. Opava, Opava, 2001, 73-84.

[3] Doubrov B., Komrakov B., Morimoto T., Equivalence of holonomic differential equations, Lobachevskii J. Math. 3 (1999), 39-71.

[4] Fels M., The equivalence problem for systems of second-order ordinary differential equations, Proc. London Math. Soc. 71 (1995), 221-240.

[5] Lie S., Vorlesungen über Differentialgleichungen mit bekannten infinitesimalen Transformationen, Leipzig, Teubner, 1891.

[6] Medvedev A., Geometry of third order ODE systems, Arch. Math. (Brno) 46 (2010), 351-361.

[7] Morimoto T., Geometric structures on filtered manifolds, Hokkaido Math. J. 22 (1993), 263-347.

[8] Sato H., Yoshikawa A.Y., Third order ordinary differential equations and Legendre connections, J. Math. Soc. Japan 50 (1998), 993-1013.

[9] Tanaka N., On differential systems, graded Lie algebras and pseudo-groups, J. Math. Kyoto. Univ. 10 (1970), 1-82.

[10] Tanaka N., On the equivalence problems associated with simple graded Lie algebras, Hokkaido Math. J. 8 (1979), 23-84.

[11] Tresse M.A., Détermination des invariants ponctuels de l'équation différentielle ordinaire du second ordre $y^{\prime \prime}=\omega\left(x, y, y^{\prime}\right)$, Leipzig, Hirzel, 1896.

[12] Yano K., The theory of Lie derivatives and its applications, North Holland Publishing Co., Amsterdam, 1957. 